\title{
Evaluation of Video Quality Based on Objectively Estimated Metric
}

\author{
Harilaos Koumaras, Anastasios Kourtis, and Drakoulis Martakos
}

\begin{abstract}
Multimedia applications, and especially encoded video services, are expected to play a major role in the 3rd generation (3G) and beyond mobile communication systems. Given that future service providers are expected to provide video applications at various price and quality levels, quick and economically affordable methods for preparing/encoding the offering media at various qualities are necessary to be developed. This paper presents a method for objective evaluation of the perceived quality of MPEG4 video content, based on a quantification of subjective assessments. Showing that subjectively derived perceived quality of service $(\mathrm{PQoS})$ vs. bit rate curves can be successfully approximated by a group of exponential functions, the proposed method exploits a simple objective metric, which is obtained from the mean frame rate vs. bit rate curves of an encoded clip. The validity of this metric is assessed by comparing subjectively derived $\mathrm{PQOS}$ results to the corresponding ones, which come from the proposed objective method, showing that the proposed technique provides satisfactory PQoS estimation.
\end{abstract}

Index Terms: MPEG-4, objective measurements, perceived quality of service (PQoS).

\section{INTRODUCTION}

The mobile communication systems of the 3rd generation $(3 \mathrm{G})$, among which UMTS is the most dominant, will provide the platforms for a multitude of novel services and applications. Multimedia-enriched services, including Internet access, downloading, and streaming of short video clips, etc., are expected to lead in usage, operator revenue and bandwidth consumption in mobile networks. The delivery of on-demand mobile media will enable many powerful services (like video telephony and playback of short video clips, etc.), making even more important the need for quality of service in $3 \mathrm{G}$ networks.

One of the $3 \mathrm{G}$ visions is that multimedia services will be sold in a consumer mass market based on the provision of content at a requested quality [1]. There are a number of approaches to this, one being the use of perceived quality of service (PQoS) concept. The evaluation of the PQoS for multimedia and audiovisual content that have variable bandwidth demands will provide a user with a range of choices covering the possibilities of

Manuscript received October 17, 2003; approved for publication by Hanseok Ko, Division I Editor, April 20, 2005.

H. Koumaras is with the University of Athens, Informatics and Telecommunication Department, Panepistimioupoli, Zografou, 15789, Athens, Greece, email: koumaras@di.uoa.gr, koumaras@iit.demokritos.gr.

A. Kourtis is with the Institute of Informatics and Telecommunications NCSR “Demokritos,” Patriarchou Gregoriou Str., Agia Paraskevi, Attiki, 15310, Athens, Greece, email: kourtis@iit.demokritos.gr.

D. Martakos is with the University of Athens, Informatics and Telecommunication Department, Panepistimioupoli, Zografou, 15789, Athens, Greece, email: martakos@di.uoa.gr. low, medium, or high quality connections, indication of service availability and cost. The future end users will be able to choose the QoS level that they want to start the session with, so that the service provider can launch the service with a set of parameters that match those requested by the user [1].

The application of the PQoS concept gives the operator (or even the service provider) the capability of a better exploitation of the network resources for delivering multimedia applications (like MMS-video) to the users, because it is possible to allocate only those radio resources sufficient to maintain user satisfaction. Since this is true for each user, then the overall allocation of the radio resource results in an optimization of the system spectral efficiency.

The information society technologies (IST) project Enthrone (FP6-507637) aims to promote the adoption and usage of the perceived quality of service (PQoS) concept in upcoming $3 \mathrm{G}$ networks. In this context, one of its major objectives is to develop efficient and cost-effective solutions for the delivery of the envisaged services at a requested (user perceived) quality. This is essential for those services targeting a consumer mass market (existing and new QoS-based interactive services) and will in turn justify the investment to such services and technologies for the business entities involved in a 3G (and beyond) venture.

In order to provide audiovisual content at different perceived quality levels, it is necessary to define the variation of PQoS as an equation of the encoding bit rate. Precise curves of PQoS vs. bit rate can be derived using an audience of people, who are watching a short video clip and score its quality, as perceived by them. However, this procedure is expensive and time consuming. For actual commercial applications, objective measurement of PQoS for any type of video content is required. In this respect, this paper defines a simple metric for the measurement of the PQoS level and presents a method that is capable of making objective measurements of the perceived quality of audiovisual content, in the form of a short video clip. The results of this method can be used by a service provider in order to encode the various available on-demand media clips at bit rates, which correspond to specific quality levels. In this way, the provider achieves an optimization of the storage requirements and system bandwidth efficiency.

The rest of the paper is organized as follows. Section II presents literature review and related work in the issue of PQoS evaluation. In Section III, a quantification of subjective assessments is presented. Section IV describes the proposed objective PQoS evaluation method. Section V extends the experimental measurements to real captured video clips, proving therefore the validity of the proposed metric to no-reference clips. Section VI examines the efficiency of the proposed method by comparing subjectively derived data to the corresponding ones that result 
from the proposed method. Finally, Section VII concludes the paper.

\section{LITERATURE REVIEW AND RELATED WORK}

Lately, emphasis has been put on developing methods and techniques for evaluating the perceived quality level of video content. These methods can be mainly categorized into two major classes, the subjective and objective ones.

The subjective test methods involve an audience of people, who watch a video sequence and evaluate its quality as perceived by them, under specific and controlled watching conditions. The mean opinion score (MOS) is regarded as the most reliable method of quality measurement and has been applied on the most known subjective techniques: The single stimulus continue quality evaluation (SSCQE) and the double stimulus continue quality evaluation (DSCQE) [2]-[4]. However, the MOS method is inconvenient due to the fact that the preparation and execution of subjective tests is costly and time consuming. For this reason, a lot of effort has recently been focused on developing cheaper, faster and easier applicable objective evaluation methods. These techniques successfully emulate the subjective quality assessment results, based on criteria and metrics that can be measured objectively. The objective methods are classified, according to the availability of the original video signal, which is considered to be in high quality.

The majority of the proposed objective methods require the undistorted source video sequence as a reference entity in the quality evaluation process and due to this are characterized as full reference methods [5], [6]. These methods are based on an error sensitivity framework with most widely used metrics the peak signal to noise ratio (PSNR) and the mean square error (MSE).

However, these overused metrics have seriously been criticized that they do not provide reliable measurements of the perceived quality [13]. For this reason, a lot of effort has been focused on developing assessment methods that emulate characteristics of the human visual system (HVS) [7]-[10] using contrast sensitivity functions (CSF), channel decomposition, error normalization, weighting and finally Minkowski error pooling for combining the error measurements into a single perceived quality estimation. An analytical description of the framework, which these methods use, can be found in [11].

However, it has been reported [12], [13] that these complicated methods do not provide more reliable results than the simple mathematical measures (such as PSNR). Due to this some new full reference metrics that are based on the video structural distortion, and not on error measurement, have been proposed [14], [15].

On the other hand, the fact that these methods require the original video signal as reference deprives their use in commercial video service applications, where the initial undistorted clips are not accessible. Moreover, even if the reference clip is available, then synchronization predicaments between the undistorted and the distorted signal (which may have experienced frame loss) make the implementation of the full reference methods difficult.

Due to these reasons, the recent research has been focused on developing methods that can evaluate the PQoS based on met-

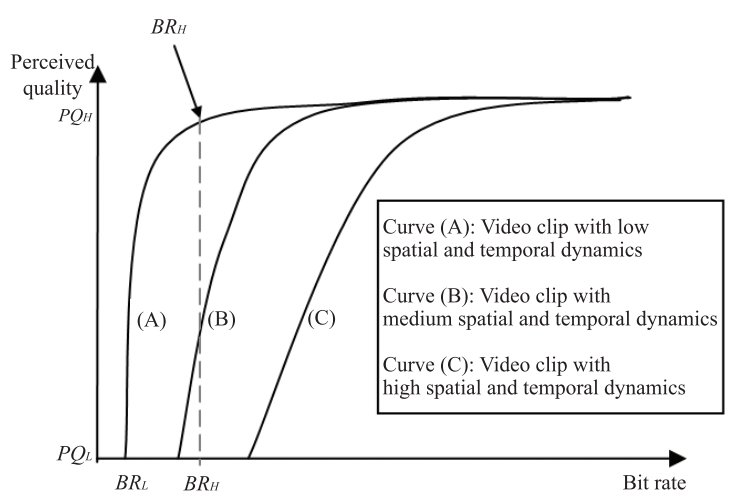

Fig. 1. PQoS vs. bit rate curves.

rics, which use only some extracted features from the original signal (reduced reference methods) [16] or do not require any reference video signal (no reference methods) [17], [18].

All the aforementioned post-encoding methods may require repeating post-encoding tests in order to determine the encoding parameters that satisfy a specific level of user satisfaction, making them time consuming, complex and impractical for implementation on the $3 \mathrm{G} / 4 \mathrm{G}$ multimedia mobile applications. Due to the fact that the $3 \mathrm{G} / 4 \mathrm{G}$ vision is the provision of audiovisual content at various quality and price levels [1], there is great need for developing methods and tools that will help service providers to predict quickly and easily the PQoS level of a media clip. These methods must be able to determine the specific encoding parameters that satisfy a certain quality level, without the need of repeating post-encoding tests and quality evaluations.

In this context, this paper presents a novel objective evaluation method, based on a quantification of subjective evaluation results, which will enable the quick and easy estimation of the PQoS level for MPEG-4 coded video clips, alleviating therefore the time requirements of the already existing methods.

\section{DEFINITION OF METRICS FOR EVALUATING PQOS}

Among the standardized digital video encoding formats (MPEG-1/2/4), the MPEG-4 [19] is mostly preferred in the distribution of interactive multimedia services over IP, while MPEG-2 is almost exclusively used in DVB networks. Furthermore, MPEG-4 is most suitable for $3 \mathrm{G}$ networks, because it provides better encoding efficiency at low bit rates, compared to the other two formats, given that UMTS can reach up to 2 Mbit/sec maximum bit rate. The most reliable method to measure the perceived quality level of audiovisual content is to use subjective evaluations. Curves of PQoS vs. bit rate can be derived using an audience of people, who are watching a short video clip and score its quality, as perceived by them [4], [20][23]. Such curves are shown in Fig. 1. Curve (A) represents a video clip with low temporal and spatial dynamics, whose content has "poor" movements and low picture complexity. Curve (C) represents a short video clip with high dynamics. Curve (B) represents an intermediate case. According to these subjective experiments, it can be derived that when the encoding bit rate 
drops below a certain threshold, which is depended on the video content, then the quality practically collapses.

Moreover, the quality improvement is not significant for bit rates higher than a specific threshold, which is also dependent on the spatial and temporal activity of the clip. Finally, it is derived that the variation of the quality level versus the encoding bit rate is a non linear increasing function, with slope depending on the video dynamics. Thus, it is evident that video dynamics play a major role in the shape of the PQoS vs. bit rate curve. Furthermore, as more than one video may have similar dynamics, each curve corresponds to a family of videos with related characteristics (dynamics).

This paper proposes that each PQoS vs. bit rate curve can be characterized by (a) the low bit rate $\left(B R_{L}\right)$, which corresponds to the lowest value of the accepted PQoS $\left(P Q_{L}\right)$ by the audience, (b) the high bit rate $\left(B R_{H}\right)$, which corresponds to the minimum value of the bit rate for which the PQoS reaches its maximum value $\left(P Q_{H}\right)$ (see $B R_{H}$ for curve (A) in Fig. 1), and (c) the shape and subsequently the inclination of each curve. From the curves of Fig. 1, it can be deduced that video clips with low dynamics have lower $B R_{L}$ and higher inclination than clips with high dynamics.

A general analytical expression for the curves in Fig. 1 has not yet been derived and there is still work to be done in theoretical level. Furthermore, the fact that these curves are derived from subjective tests deprives their use in practical and commercial systems. A simplified approach to these curves, based on metrics that can be derived objectively, would be very useful for applications in commercial systems.

The method proposed in this paper is based on an exponential approximation of the curves of Fig. 1. Each curve can be approximately described by an equation of the form

$$
\begin{gathered}
P Q=\left(P Q_{H}-P Q_{L}\right)\left(1-e^{-a\left(B R-B R_{L}\right)}\right)+P Q_{L}, \\
B R>B R_{L} \text { and } \alpha>0
\end{gathered}
$$

where $P Q$ is the perceived quality level of a specific video clip at bit rate $B R$, with $P Q_{H}$ and $P Q_{L}$ being the maximum and minimum quality levels, respectively. The parameter $\alpha$ defines the shape and subsequently the inclination of each curve.

From (1), it is evident that the $P Q$ level of a specific video clip can be defined, provided that $B R_{L}$ and parameter $\alpha$ are known (considering that $P Q_{H}$ remains practically constant for all the test videos). In this respect, in the following sections a correlation between $B R_{L}$ and parameter $\alpha$ is derived, which entails that the objective evaluation of only one metric (i.e., $B R_{L}$ ) is sufficient to estimate the various PQoS levels of a video clip.

\section{OBJECTIVE ESTIMATION OF $B R_{L}$ AND CORRELATION WITH PARAMETER $\alpha$}

For the needs of the proposed method, five test video clips with different content dynamics each, were used. Their initial encoding format was MPEG-2/PAL at $12 \mathrm{Mb} / \mathrm{s}$. These well known video clips have been developed by Tektronix corp. and Sanoff laboratories and are shown on Table 1. Each test video clip was transcoded from its original MPEG-2/PAL format at $12 \mathrm{Mb} / \mathrm{s}$ to DivX MPEG-4 format, at various constant bit rates
Table 1. The five test video clips.

\begin{tabular}{|c|c|c|}
\hline Clip 1 & \multirow{2}{*}{$\begin{array}{c}\text { Low } \\
\text { dynamics }\end{array}$} & Suzie \\
\cline { 1 - 1 } Clip 2 & $\vdots$ & Cactus \& Comb \\
\cline { 1 - 1 } Clip 3 & $\vdots$ & Table tennis \\
\cline { 1 - 1 } Clip 4 & High & Flower garden \& Mile \\
\cline { 1 - 1 } Clip 5 & dynamics & Mobile \& Calendar \\
\hline
\end{tabular}

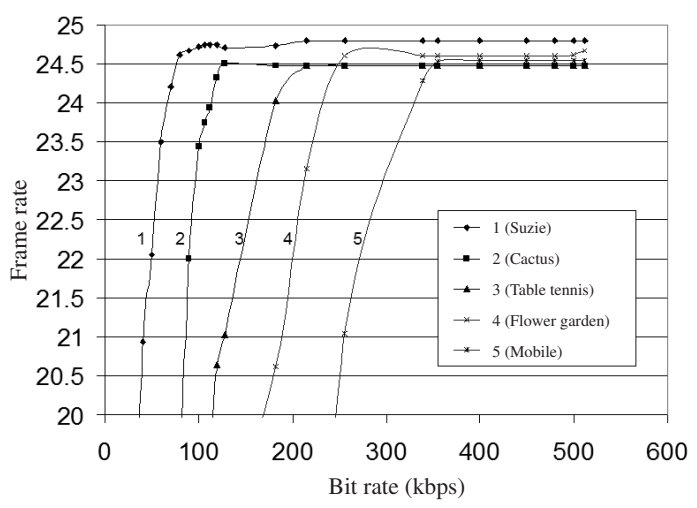

Fig. 2. The mean fps vs. bit rate curves for CIF resolution and $80 \%$ smoothness parameter.

(CBR) from $50 \mathrm{kbps}$ to $500 \mathrm{kbps}$. For each bit rate, a different MPEG-4 file was created. CIF (common intermediate format) resolution $(352 \times 288)$, smoothness parameter equal to $80 \%$ and constant frame rate with 25 frames per second (fps) were common parameters for the transcoding process in all test videos.

Each MPEG-4 video clip was then played back using a suitable Microsoft media player, featuring an option to measure the mean value of fps over the whole duration of the video clip (statistics option). During playback, it was observed that the perceived quality decreases as the bit rate drops, even though the mean frame rate remains constant to the initial value (i.e., 25 fps for the experiments of this paper), as measured by the player statistics tool. It was also observed that when the bit rate drops below a certain threshold, picture pauses appear. These can be measured by the mean frame rate, which drops below the initial value ( $25 \mathrm{fps}$ ), as measured by the statistics tool.

The variation of the mean frame rate during playback, versus encoding bit rate is shown in Fig. 2, for the various test video clips. Note that since the statistics tool measures mean values, the points on the curves do not correspond always on integer values. The content of each video clip and its corresponding numbering is shown in Table 1. Clips $1,2, \cdots, 5$ are listed in ascending order, concerning the dynamics of their video content. From Table 1 and the curves of Fig. 2, it is evident that the value of the bit rate, below which the mean frame rate drops under its original value ( $25 \mathrm{fps}$ ), depends on the video content dynamics of the clip. In other words, video clips with high dynamics (e.g., Mobile \& Calendar) require higher encoding bit rate to maintain the mean fps value at its initial value (i.e., 25), than video clips with lower dynamics (e.g., Suzie).

In order to correlate curves of Figs. 1 and 2, the following assumption can be made. When the frame rate drops below 25 fps, the perceived quality is considered to be unacceptable. So, it is possible to consider that the $B R_{L}$ value of each curve in 


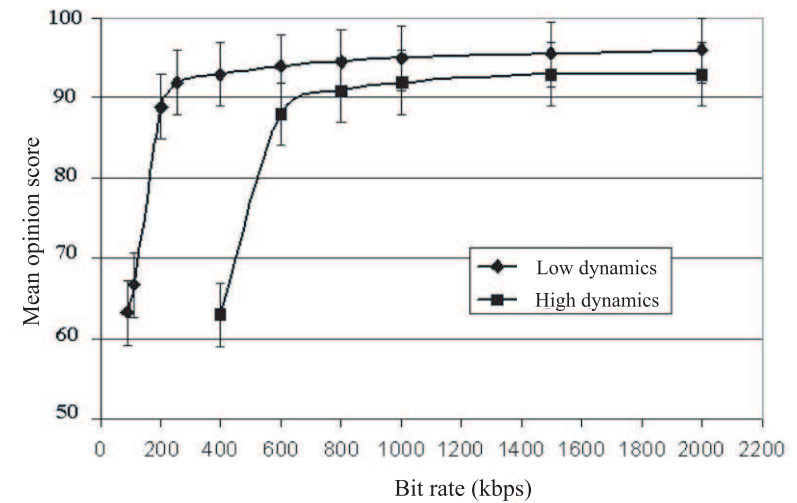

Fig. 3. Mean opinion score vs. bit rate derived from subjective test for videos with high and low dynamics.

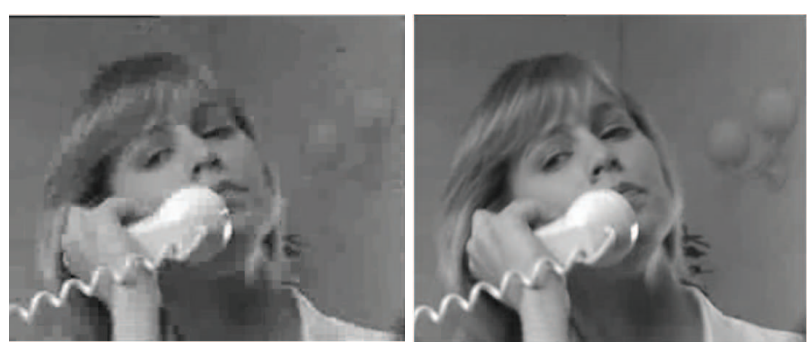

Fig. 4. Comparison between two frames from the test signal "Suzie" encoded with MPEG-4/CIF at $B R_{L}=90 \mathrm{kbps}$ and $B R_{H}=2.5 B R_{L}=$ 225 kbps.

Fig. 1 is equal to the objectively measured bit rate in Fig. 2, where the curve starts to bend. Both values correspond to the minimum level of accepted perceived quality $\left(P Q_{L}\right)$.

Therefore, this paper proposes that $B R_{L}$ can be objectively defined by the encoding bit rate, below which the target-initially selected frame rate $(25 \mathrm{fps})$ cannot be achieved. This means that for lower bit rates than $B R_{L}$, the frame rate drops below its initial value. The explanation of this phenomenon lies on the fact that the rate algorithm of the encoder is not able to achieve the target low bit rate value by altering only the quantization scale. Therefore, the encoder is forced to exclude some frames from the encoding process. By this way the total information is minimized, and the target low bit rate can be successfully achieved. The bit rate, at which the frame loss begins, depends on the spatial and temporal complexity of each video sequence. This observation is reconfirmed by the experimental results of Fig. 2, where the frame loss for high dynamics clips occurs at higher bit rates in comparison to low dynamics clips. Although in MPEG4 encoders, it is possible to select the encoding frame rate (typically 10 to $30 \mathrm{fps}$ ), for reference reasons, the frame rate that was used for all the test signals in this paper, was equal to $25 \mathrm{fps}$.

In order to specify the shape of an exponential curve, like the ones in Fig. 1, it is sufficient to define one point of the curve and parameter $\alpha$ (see (1)). This point can be $\left(B R_{L}, P Q_{L}\right)$, where $P Q_{L}$ is the lowest acceptable perceived quality level. Parameter $\alpha$ can be defined using a second point of the curve, which can be selected to be $\left(B R_{H}, P Q_{H}\right)$, where $P Q_{H}$ the maximum achieved quality level. The value of $B R_{L}$ can be objectively

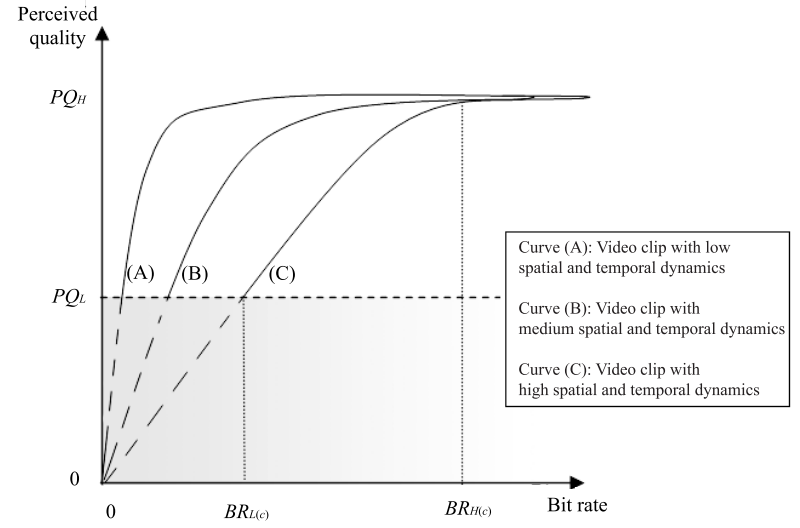

Fig. 5. Theoretical approach of equation $B R_{H}=2.5 B R_{L}$.

measured, as explained above. The value of $B R_{H}$ can be obtained indirectly, from $B R_{L}$.

In order to define the relation between $B R_{L}$ and $B R_{H}$, a subjective evaluation process was used. In this respect, an audience of ten people was asked to watch and score twenty high dynamics and twenty low dynamics video clips, which were encoded at various constant bit rates with DivX MPEG-4/CIF format and constant frame rate equal to $25 \mathrm{fps}$. Encoding bit rates were varied from 80 to $2000 \mathrm{kbps}$, depending on the video dynamics. Fig. 3 illustrates the mean opinion score (MOS) vs. the encoding bit rate for the quality assessment of the twenty high and low dynamics test signals, respectively. The scale that was used in these subjective evaluation experiments, is the DSCQS ITU$\mathrm{R}$ [3], [22] hundred scale, which considers best possible quality $P Q_{H}=100$ (i.e., excellent quality) and lowest acceptable quality level $P Q_{L}=60$ (i.e., fair quality).

From Fig. 3, it can be verified that, when the bit rate exceeded a specific threshold (1000 kbps for high dynamics and 250 for low dynamics clips), the viewers assessed that the quality is not substantially improved any more. Given this and provided that the value of $B R_{L}$ was around $400 \mathrm{kbps}$ and $90 \mathrm{kbps}$, for high and low dynamics videos, respectively, it can be deduced that when the ratio $B R_{H} / B R_{L}$ equals to 2.5 , the quality has approximately reached satisfactorily its best quality level. Fig. 4, depicts two representative frames derived from the test sequence Suzie, encoded at $B R_{L}=90 \mathrm{kbps}$ and $B R_{H}=2.5 B R_{L}=225$ kbps with MPEG-4/CIF, from where the substantial quality improvement can be observed.

The above experimentally derived expression of $B R_{L}$ and $B R_{H}$ (i.e., $B R_{H}=2.5 B R_{L}$ ) can be theoretically justified, based on the proposed exponential approximation of PQoS vs. bit rate curves. Considering that the curves of Fig. 1 can be extended to values below $P Q_{L}$ (i.e., to unacceptable/annoying quality levels), Fig. 5 can be derived. It is assumed that, theoretically, all curves converge to $P Q=0$, although the gray area (dashed lines) is not acceptable for actual services provision. From this figure, it can be deduced that the subjectively derived PQoS vs. bit rate curves can be represented as segments of an exponential curves family, which can be described by the 


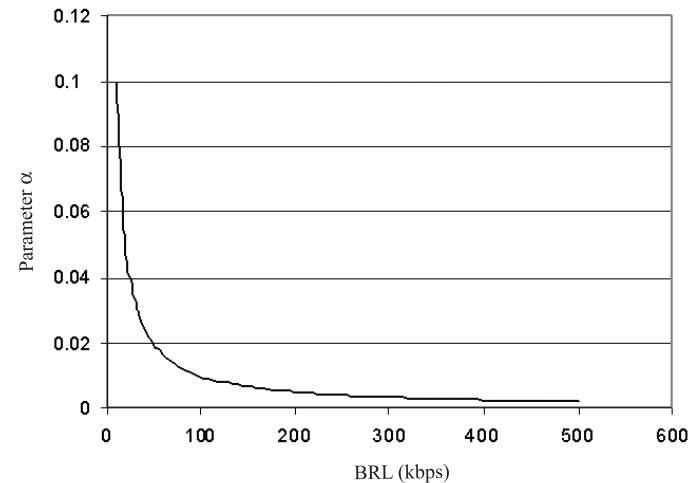

Fig. 6. Parameter $\alpha$ vs. BRL.

expression

$$
P Q=P Q_{H}\left(1-e^{-\alpha B R}\right)
$$

where only the parameter $\alpha$, and subsequently the slope, changes according to the video dynamics. Therefore, (2) covers all the possible PQoS curves that may be derived from any group of clips with low, medium or high spatial and temporal activity level. Equation (2) can be directly derived from (1) without loss of generality by setting $P Q_{L}=0$ and $B R_{L}=0$.

From (2), the $B R_{L}$ value, which corresponds to $P Q_{L}$, can be expressed as

$$
B R_{L}=\frac{\ln \frac{P Q_{H}}{P Q_{H}-P Q_{L}}}{\alpha} .
$$

Thus, replacing in (2) the $B R$ value with the expression $k \cdot B R_{L}$, it is deduced that

$$
P Q=P Q_{H}\left[1-\left(\frac{P Q_{H}-P Q_{L}}{P Q_{H}}\right)^{k}\right] .
$$

Finally, replacing in (4) $P Q_{H}=100, P Q_{L}=60$, and $k=2.5$, which are the test parameters of the subjective evaluation procedures in this paper, it is deduced that when $B R=2.5 B R_{L}$, then the derived PQoS level approximates satisfactorily the maximum perceived quality level at a percentage of $90 \%$. Moreover, solving (3) by $\alpha$, it can be derived that

$$
\alpha=\frac{\ln \frac{P Q_{H}}{P Q_{H}-P Q_{L}}}{B R_{L}} .
$$

Replacing in (5) $P Q_{H}=100$ and $P Q_{L}=60$, it is deduced that

$$
\alpha=\frac{0.92}{B R_{L}} .
$$

Fig. 6 illustrates the graphical representation of (6).

Therefore, it is evident that the determination of the exponentially approximated curve of PQoS vs. bit rate for a specific video clip is possible, by first finding the value of $B R_{L}$ from the corresponding curve of fps vs. bit rate (Fig. 2) and then using this $B R_{L}$ value as input to (6), in order to find the corresponding value of parameter $\alpha$. These two values (i.e., $B R_{L}$ and parameter $\alpha$ ) are sufficient to define the corresponding exponential (1)

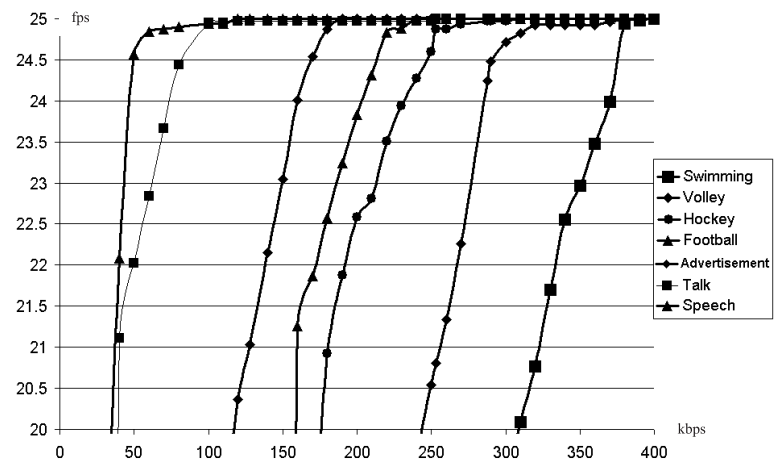

Fig. 7. Mean fps vs. kbps for actual/non-reference video clips (CIF resolution).

and ultimately define the bit rates that correspond to the various quality levels.

Referring to the curves of Fig. 1, it is obvious that the number of the significantly different quality levels depends on the dynamics of the video clip. Using a typical scale of PQoS, a low dynamic video clip will have short distance $\left[B R_{L}, B R_{H}\right]$ and therefore it is meaningless to offer the specific video clip at a lot of quality levels. Correspondingly, for a high dynamic video clip the offering quality levels will be many. So, it is up to the service provider to determine about the intermediate quality levels of a video clip, based on the bit rates that correspond to the lowest and highest quality levels, which are defined by the proposed method.

\section{EXTENSION OF THE PROPOSED METHOD}

The proposed method was also tested on actual video clips with various dynamics. The encoding format of these clips was MPEG-4 with $352 \times 288$ resolution (CIF) and 25 fps. Fig. 7 depicts the mean fps vs. the encoding bit rate for video clips, captured from a typical satellite TV program. From these figures, it is obvious that video clips with high dynamics (like swimming/sports) appear to have $B R_{L}$ values higher than video clips with low dynamics (like talk show). So, it is evident that the metric $B R_{L}$ is able to distinguish actual video content according to its dynamics.

The frame rates vs. bit rate curves were also derived for the five reference signals (Table 1) for the case of QCIF resolution. Fig. 8 shows the corresponding $B R_{L}$ values of the five reference videos, when encoded with CIF and QCIF resolution, respectively.

From Fig. 8, it can be deduced that the reduction of $B R_{L}$ is more than $50 \%$ when QCIF is used instead of CIF resolution. Provided that in the upcoming $3 \mathrm{G}$ mobile communication networks (UMTS) the user terminals will be equipped with small color screens (supporting QCIF resolution) and provided that the bit rate must be kept in significant low levels, it is obvious that QCIF resolution will dominate in the distribution of multimedia files in $3 \mathrm{G}$ mobile devices. To this direction the proposed method aims to contribute, as a part of real commercial applications.

Therefore, the objectively measured metric $B R_{L}$ is able to 


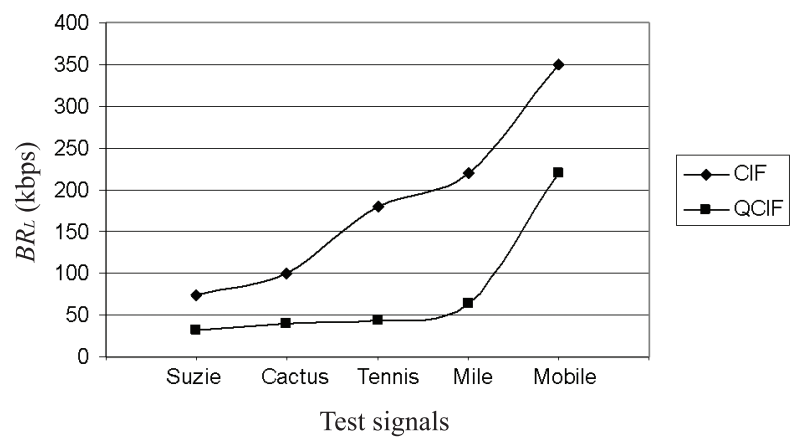

Fig. 8. The values of $B R_{L}$ for CIF and QCIF resolution.

quantitatively estimate the perceived quality of a video content. This metric applies for both CIF and QCIF resolution and its validity has been successfully tested not only for reference video clips, but for actual TV clips, as well. The proposed method can be used for the provision of video content at different quality levels (and corresponding prices), a feature, which is considered significant in the provision of multimedia services in the upcoming $3 \mathrm{G}$ networks.

\section{EFFICIENCY EVALUATION OF THE PROPOSED METHOD}

This section examines the efficiency of the proposed method. In this respect, it was performed a comparison between the quality evaluation data derived from subjective procedures and the data, which the proposed method provides. Regarding the efficiency evaluation, two separate tests were performed, where it was examined the efficiency of the proposed objective metric $\left(B R_{L}\right)$ and the efficiency of the proposed exponential approximation method for estimating the PQoS level of an encoded video clip.

\section{a. Efficiency evaluation of the objectively estimated $B R_{L}$}

In order to examine the validity of the objectively estimated $B R_{L}$ metric, the five reference video clips of Table 1 were used in CIF resolution. For these five reference signals, the objectively estimated $B R_{L}$ values were derived (Table 2 ) from the corresponding fps vs. bit rate curves, which are depicted in Fig. 2. Furthermore, the same test signals were used as test bed in a subjective evaluation procedure, where the audience was asked to select the encoded video with the lowest acceptable quality level (i.e., tilling artifacts may appear but the video motion should be smooth, without pauses and interruptions). By this way the corresponding $B R_{L}$ values were estimated subjectively. The results are depicted in Table 2 in conjunction with the corresponding objective ones.

Based on these results, it can be derived that the proposed metric provides accurate estimations and emulates successfully the corresponding subjective evaluation results with a worst case error equal to $10 \%$.

b. Efficiency evaluation of the proposed exponentially based method for estimating the PQoS vs. bit rate curve

The efficiency of the proposed method for predicting the PQoS level of a test signal, was examined by comparing the
Table 2. The subjectively and objectively estimated BRL for the four test signals.

\begin{tabular}{|c|c|c|}
\hline Test signal & $\begin{array}{c}\text { Objectively estimated } \\
B R_{L}(\mathrm{kbps})\end{array}$ & $\begin{array}{c}\text { Subjectively estimated } \\
B R_{L}(\mathrm{kbps})\end{array}$ \\
\hline Suzie & 75 & 80 \\
\hline Cactus & 100 & 100 \\
\hline Table tennis & 180 & 170 \\
\hline Flower garden & 220 & 200 \\
\hline Mobile \& Calendar & 350 & 380 \\
\hline
\end{tabular}

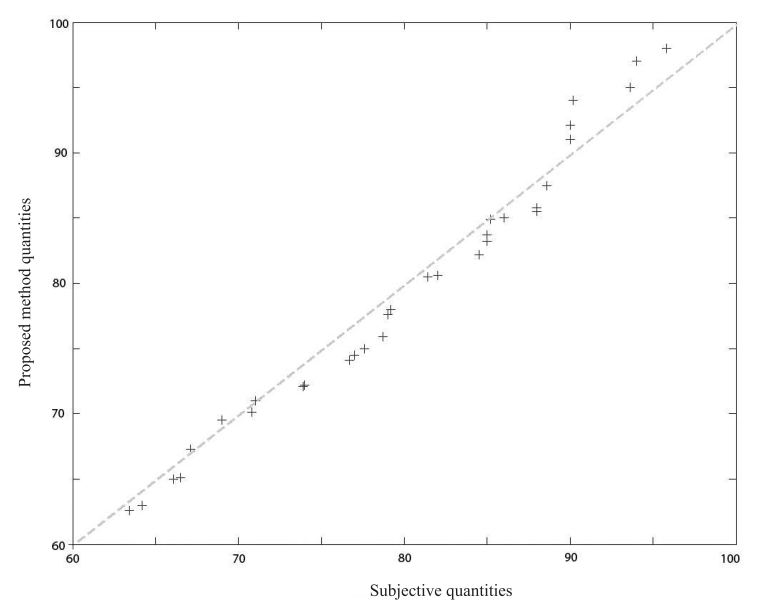

Fig. 9. The $Q-Q$ plot of the subjectively and objectively derived data.

quality evaluation results derived from a subjective evaluation process to the corresponding ones, which came from the proposed method. In this respect, an audience of ten people was asked to watch and evaluate the quality of five test signals, containing various TV captured dynamic contents (ranging from static to very active video contents). The test sequences were encoded from their original digital video (DV) format to DivX MPEG-4/CIF format at various constant bit rates (ranging from 80 to $1500 \mathrm{kbps}$ ) and $25 \mathrm{fps}$. The total number of the encoded test sequences was 35 . Finally, the MOS for each encoded sequence was derived from the statistical process of the individual evaluations.

Afterwards, the proposed objective method was applied on the test signals that were used in this subjective evaluation process. By calculating objectively the corresponding $B R_{L}$ values from the frame rate vs. bit rate curves and making use of the relation (6), then the determination of the (1) for each video clip was possible. Finally, the quality levels that correspond to each encoded bit rate, which was used in the subjective test, could be easily and quickly estimated, by simply solving (1).

Having derived both the subjective and objective scores for each encoded test signal, a Q-Q plot was drawn, in order to examine the efficiency of the proposed method. A Q-Q plot is illustrated based on values, which have been derived from two samples, and examines whether these two samples come from the same distribution type. If the samples do come from the same distribution then the plot is linear.

Fig. 9 depicts the Q-Q plot for the subjectively and objectively derived data for all the encoded sequences. As it can be 
observed, the distribution of the points is around and very close to the linear diagonal, which demonstrates the satisfactory fit of the two sets. Therefore, the proposed method of this paper provides, quickly and easily, reliable results of PQoS evaluation for MPEG-4 coded clips. In this respect, the proposed method can be commercially used and applied in the upcoming 3G/4G mobile communication systems, where multimedia services will be offered at multiple quality levels.

\section{CONCLUSIONS}

The provision of audiovisual content at different perceived quality levels requires curves of PQoS vs. bit rate, which are usually derived via subjective methods, using an audience of people. This paper defines a metric for the quantitative estimation of the PQoS level and presents a method that is capable of making objective estimations of the perceived quality of audiovisual content. Showing that subjectively derived PQoS vs. bit rate curves can be successfully approximated by a group of exponential functions, the efficiency of the proposed technique is tested and proved that provides quick and efficient PQoS estimation, emulating successfully the corresponding subjective evaluation results. A series of experiments was also conducted with real video content, captured from common TV programs, showing that the proposed method can successfully estimate the PQoS level for encoded video clips in both CIF and QCIF formats. Given that in $3 \mathrm{G} / 4 \mathrm{G}$ mobile communication systems, the multimedia services will be offered at various perceived quality levels, the proposed method can be valuable in $3 \mathrm{G} / 4 \mathrm{G}$ mobile communication applications.

\section{ACKNOWLEDGMENTS}

The work in this paper was carried out in the frame of the information society technologies (IST) project Enthrone (FP6507637).

\section{REFERENCES}

[1] P. Seeling, M. Reisslein, and B. Kulapala, "Network performance evaluation using frame size and quality traces of single layer and two layer video: A tutorial," IEEE Commun. Surveys, vol. 6, no. 3, 3rd Quarter 2004.

[2] T. Alpert and L. Contin, "DSCQE experiment for the evaluation of the MPEG-4 VM on error robustness functionality," ISO/IEC-JTC1/SC29/ WG11, MPEG 97/M1604, 1997.

[3] ITU-R, "Methodology for the subjective assessment of the quality of television pictures," Recommendation ITU-R BT.500-7, 2000.

[4] F. Pereira and T. Alpert, "MPEG-4 video subjective test procedures and results," IEEE Trans. Circ. Syst. Video Technol., vol. 7 (1), pp. 32-51, 1997.

[5] K. T. Tan and M. Ghanbari, "A multi-metric objective picture quality measurements model for MPEG video," IEEE Trans. Circ. Syst. Video Technol., vol. 10 (7), pp. 1208-1213, 2000.

[6] St. Wolf and M. H. Pinson, "Spatial-temporal distortion metrics for inservice quality monitoring of any digital video system," in Proc. SPIE Int. Symp. Voice Video Data Commun., Boston, MA, Sept. 11-22, 1999.

[7] A. B. Watson, J. Hu, and J. F. McGowan, "DVQ: A digital video quality metric based on human vision," J. Electron. Imaging, vol. 10 (1), pp. 2029, 2001.

[8] S. Daly, "The visible difference predictor: An algorithm for the assessment of image fidelity," in Proc. SPIE'92, vol. 1616, 1992, pp. 2-15.

[9] A. P. Bradley, "A wavelet difference predictor," IEEE Trans. Image Processing, vol. 5, pp. 717-730, 1999.
[10] Y. K. Lai and J. Kuo, "A Haar wavelet approach to compressed image quality measurement," J. Visual Commun. Image Understanding, vol. 11, pp. 81-84, 2000.

[11] Z. Wang, H. R. Sheikh, and A. C. Bovik, Objective video quality assessment in The Handbook of Video Databases: Design and Applications, B. Furht and O. Marqure, eds., CRC Press, pp. 1041-1078, 2003.

[12] VQEG, "Final report from the video quality experts group on the validation of objective models of video quality assessment," 2000.

[13] Z. Wang, A. C. Bovik, and L. Lu, "Why is image auality assessment so difficult," in Proc. IEEE ICASSP 2002, vol. 4, 2002, pp. 3313-3316.

[14] Z. Wang, L. Lu, and A. C. Bovik, "Video auality assessment based on structural distortion measurement," Sig. Processing: Image Commun., special issue on "Objective video quality metrics," vol. 19 (2), pp. 121-132, 2004.

[15] Z. Wang, A. C. Bovik, H. R. Sheikh, and E. P. Simoncelli, "Image quality assessment: From error visibility to structural similarity," IEEE Trans. Image Processing, vol. 13 (4), pp. 1-14, 2004.

[16] I. P. Guawan and M. Ghanbari, "Reduced-reference picture quality estimation by using local harmonic amplitude information," in Proc. London Commun. Symp. 2003, 2003.

[17] L. Lu, Z. Wang, A. C. Bovik, and J. Kouloheris, "Full-reference video quality assessment considering structural distortion and no-reference quality evaluation of MPEG video," in Proc. IEEE Int. Conf. Multimedia, 2002

[18] J. Lauterjung, "Picture quality measurement," in Proc. Int. Broadcasting Convention (IBC'98), Amsterdam, 1998, pp. 413-417.

[19] MPEG, ISO-IEC/JTC1/SC29/WG11 N4668, “MPEG-4 overview," Mar. 2002.

[20] MPEG, ISO-IEC/JTC1/SC29/WG11 N2604, "Report of the formal verification tests on MPEG-4 video error resilience," 1999.

[21] R. P. Aldridge, D. S. Hands, D. E. Pearson, and N. K. Lodge, "Continuous assessment of digitally-coded television pictures," IEE Proc. Vision, Image, Sig. Processing, vol. 145 (2), pp. 116-123, 1998.

[22] F. Pereira and T. Ebrahimi, The MPEG-4 Book, IMSC Press Multimedia Series, pp. 669-705, 2002

[23] W. Lee and J. Srivastava, "An algebraic QoS-based resource allocation model for competitive multimedia applications," Int. J. Multimedia Tools and Appl., Kluwer Editions, vol. 13, pp. 197-212, 2001.

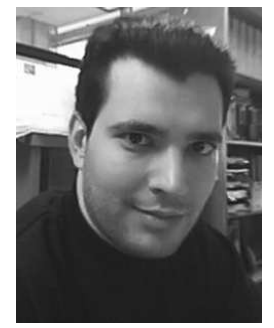

Harilaos Koumaras was born in Athens, Greece in 1980. He received his B.Sc. degree in Physics in 2002 and his M.Sc. in Electronic Automation and Information Systems in 2004, both from the University of Athens. He joined the Digital Telecommunications Lab at National Centre of Scientific Research "Demokritos" in 2003 and since then he has participated in the EU funded projects SOQUET and ENTHRONE, with presentations and publications at international conferences and book chapters. Currently, he is working toward the Ph.D. degree. His research interests include objective evaluation of the perceived quality of multimedia services, picture quality evaluation, video traffic modeling, digital terrestrial television, and video compression techniques. He is a student member of IEEE.

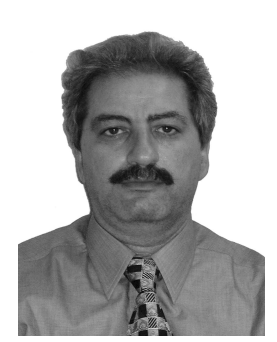

Anastasios Kourtis was born in Athens, Greece in 1956. He received his B.S. degree in Physics in 1978 and his Ph.D. degree in Telecommunications in 1984, both from the University of Athens. Since 1986, he is a researcher in the Institute of Informatics and Telecommunications of the National Centre for Scientific Research "Demokritos," currently ranking as Senior Researcher. His current research activities include digital modulation techniques, spread spectrum systems, broadband wireless networks, digital terrestrial interactive television, satellite communications and perceived quality of video services. He has published extensively in the fields above, in international scientific journals, edited books and conference proceedings. Dr. Kourtis has a leading participation in many EU funded research projects from the ESPRIT (FCPN, OFSES), ACTS (CRABS, WATT), and IST programs (WIN, MAMBO, SOQUET, CREDO, REPOSIT) to the recent IST/FP6 (ENTHRONE, ATHENA) and he is also the Project Manager of the FP6/ATHENA project. 


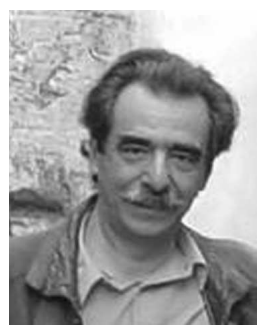

Drakoulis Martakos was born in Athens, Greece in 1947. He received his B.Sc. in Physics in 1971, M.Sc. in Electronics and Radio Communications in 1980, and Ph.D. (in the area of Real-Time Computing) in 1989, all awarded from the University of Athens, Department of Physics. He is currently an associate professor in the University of Athens, at the Department of Informatics and Telecommunications. Dr. Martakos has been employed as a consultant to various public and private organizations (ministries, the Greek Electric Company), researcher in the Laboratory of Physics of the University of Athens as well as a researcher in CERN, Geneva. He has participated in numerous national and international projects. He is author or co-author of more than 90 scientific publications and a number of technical reports and studies. 